\title{
Module function and two-way clustering analysis of Epstein-Barr virus-related nasopharyngeal cancer
}

\author{
F. Chen ${ }^{1}$, J. Liu ${ }^{1}$, M. Fan' ${ }^{2}$ X.M. Wei ${ }^{3}$, Y.L. Xie ${ }^{2}$, L.H. Wang ${ }^{1}$ and H. Yang ${ }^{1}$ \\ 'Department of Otorhinolaryngology and Head \& Neck Surgery, \\ West China Hospital, Cheng Du, Sichuan, China \\ ${ }^{2}$ Department of Otorhinolaryngology and Head \& Neck Surgery, \\ Cheng Du No. 7 People's Hospital, Cheng Du, Sichuan, China \\ ${ }^{3}$ Department of Otorhinolaryngology and Head \& Neck Surgery, \\ Chengdu 363 Hospital, Cheng Du, Sichuan, China \\ Corresponding author: H. Yang \\ E-mail: yanghuiyyhh@hotmail.com
}

Genet. Mol. Res. 13 (1): 1823-1831 (2014)

Received January 11, 2013

Accepted August 5, 2013

Published March 17, 2014

DOI http://dx.doi.org/10.4238/2014.March.17.10

\begin{abstract}
This study sought to identify and characterize the function of genes as diagnostic markers for Epstein-Barr virus (EBV)related nasopharyngeal cancer (NPC). The gene expression profile of GSE13597 was downloaded from the Gene Expression Omnibus database, which included 28 EBV-related NPC gene expression profile data sets, 25 disease samples, and 3 control samples. Data were preprocessed, and differentially expressed genes were screened using the $\mathrm{R}$ language. The co-expression coefficient was calculated to construct a co-expression network using Cytoscape. ClusterONE was used to perform module analysis to find enriched gene families. The BiCAT software was used to perform a two-way clustering analysis of differentially expressed gene expression profiles based on the coexpression networks and to verify the enriched gene families, followed by functional enrichment analysis of these gene families. The MCM
\end{abstract}


gene family was found to be enriched in EBV-related NPC. This gene family is essential for eukaryotic DNA replication. Functional analysis of differentially expressed genes in the co-expression network revealed that the enriched biological processes and pathways were mainly involved in the cell cycle. EBV-related NPC is likely associated mainly with the process of cell reproduction, providing a strong basis for the prevention, diagnosis, and treatment of EBV-related NPC and a direction for targeted chemotherapy.

Key words: Nasopharyngeal carcinoma; Co-expression networks; BiCAT; Two-way clustering

\section{INTRODUCTION}

Nasopharyngeal cancer (NPC) is a malignant tumor occurring in the nasopharyngeal cavity top and side walls. There have been 64,798 cases reported worldwide, and over $80 \%$ of these cases are from Asian countries, especially China and other southeast Asia countries (Breda et al., 2010). Epstein-Barr virus (EBV) infection and the etiology of NPC are closely related (Raab-Traub et al., 1987; Fåhraeus et al., 2006; Liu et al., 2011). EBV has been found in the majority of keratinizing squamous cell carcinoma and almost all undifferentiated squamous cell carcinoma. Because the pathogenesis of NPC is concealed and the treatment is complicated and repeated, it is important to diagnose NPC risk factors, screen high-risk populations, and perform early intervention to reduce the incidence of NPC; meanwhile, it is essential to explore NPC pathophysiology and drug intervention.

Previous studies used initial and final NPC gene signatures to uncover potential drugs with cytotoxicity to NPC cells. These studies indicated 24 upregulated and 6 downregulated bottleneck genes for predicting NPC (Lan et al., 2010). Hitt et al. (1989) generated a large cDNA library, analyzed for EBV gene expression in nude mice, and found that a family of cytoplasmic RNAs was transcribed in an antisense direction to a number of existing open reading frames in the EBV genome. Shu et al. (2006) constructed a cDNA library of NPC and obtained NPC-associated and NPC-specific antigens that elicited the immune response. Multiple chromosomal abnormalities (e.g., copy number changes on chromosomes 3p, 9p, 11q, 12p, and 14q), gene alterations (e.g., p16 deletion and LTBR amplification), and epigenetic changes (e.g., RASSF1A and TSLC1 methylation) have been identified using various genome-wide approaches (Lo et al., 2012). Consistent associations with EBV-related NPC have been reported for a handful of genes, including immune-related HLA class I genes, DNA repair gene RAD51L1, cell cycle control genes MDM2 and TP53, and cell adhesion/migration gene MMP2 (Hildesheim and Wang, 2012). New biomarkers for NPC, including EBV DNA copy number or methylation of multiple tumor suppressor genes, which can be detected in serum and nasopharyngeal brushings, have been developed for molecular diagnosis of this tumor (Tao and Chan, 2007).

Studies of EBV-related NPC through DNA microarray analyses are limited by the need for expensive instrumentation. However, microarray studies of EBV-related NPC genes of chromosomal or cDNA libraries are comprehensive and form a foundation for array analysis. This article describes the screen of key genes and the analysis of gene functions using EBV-related NPC gene expression microarray data. 


\section{MATERIAL AND METHODS}

\section{Affymetrix microarray}

GSE13597 (Bose et al., 2009) was downloaded from the Gene Expression Omnibus (GEO) database, with a total of 28 EBV-related NPC gene expression profile data sets, 25 disease samples, and 3 control samples. GSE13597 is based on the GPL96 [HG-U133A] Affymetrix Human Genome U133A Array platform. Probe annotation information was from Affymetrix, including all Affymetrix ATH1 (25 K) chip probe information, the original files, and the probe annotation information files of the platform.

\section{Data pre-processing and analysis of differentially expressed genes}

The original data were pre-processed by the Affy (Troyanskaya et al., 2001) package of the $\mathrm{R}$ language. The expression profile of 25 disease samples and 3 control samples was differentially analyzed by the limma (Fujita et al., 2006) package of the R language, and a multiple testing correction, known as the false-discovery rate (FDR), was performed by a Bayesian method; $\mathrm{P}<$ 0.05 and $|\log \mathrm{FC}|>1$ were defined as the threshold for differentially expressed genes.

\section{Prediction of interactions between proteins}

All known protein-protein interactions described in HPRD (Benjamini and Hochberg, 1995) and BIOGRID (Keshava Prasad et al., 2009) databases were obtained: 39,240 proteinprotein interactions from the HPRD database, and 326,119 protein-protein interactions from the BIOGRID database. After data integration of these 2 databases, 326,119 protein-protein interactions were collected, and a co-expression network was constructed based on these interactions.

\section{Constructing a co-expression network of differentially expressed genes}

The Pearson correlation coefficient $\left(\mathrm{r}_{\mathrm{xy}}\right)$ of different gene expression profiles was calculated by the Pearson Law as follows (Derrick et al., 1994):

$$
r_{x y}=\frac{n \sum x_{i} y_{i}-\sum x_{i} \sum y_{i}}{\sqrt{n \sum x_{i}^{2}-\left(\sum x_{i}\right)^{2}} \sqrt{n \sum y_{i}^{2}-\left(\sum y_{i}\right)^{2}}},
$$

where $x$ and $y$ represent the expression value of 2 genes in different chips. The closer to 1 the value of $r_{x y}$, the more similar the 2 gene expression profiles. If the value is equal to -1 , the 2 gene expression profiles are opposite. The closer to -1 the value of $r_{x y}$ is, the higher the opposite level. The closer to 0 the value of $r_{x y}$, the smaller the difference in the expression levels of the 2 genes. The co-expression network was constructed using Cytoscape (Shannon et al., 2003) with interaction partners with $\mathrm{r}_{\mathrm{xy}}>0.75$.

\section{Module enrichment analysis of co-expression network}

The ClusterONE plug-in of Cytoscape was used to analyze function modules (Shan- 
non et al., 2003). ClusterONE is designed to find densely connected and overlapping regions of the Cytoscape network. The densely connected regions of the protein-protein interaction network represent protein complexes or complex components. ClusterONE removed no-densely connected regions (usually 1 or 2 vertexes) using a bonding quality function. ClusterONE also found enriched modules based on the protein-protein interaction networks.

\section{Function and pathway-enrichment analysis of differentially expressed genes in the co-expression network}

The David software (Bader and Hogue, 2003) was used to obtain the enriched biological processes and pathways of all differentially expressed genes. Statistically significant was determined when FDR $<0.1$.

\section{Two-way clustering analysis}

To verify our conclusion, two-way clustering analysis and comparison of differentially expressed genes were conducted with different algorithms using BiCAT (Huang et al., 2009) and BiCAT_PLUS (Barkow et al., 2006). Two-way clustering analysis and comparison increased the information contained in the sample data set, including identification of additional pathogenic genes and function information.

\section{RESULTS}

\section{Screening for differentially expressed genes}

The original data were pre-processed using the Affy package of the $\mathrm{R}$ language to remove systematic bias as revealed by a deviation from the diagonal with increased intensity. The results showed that after pre-processing, the normalized expression profile data were differentially contrasted, and 900 differentially expressed genes met the difference threshold (P $<0.05$ and $|\log \mathrm{FC}|>1)$. The final data set contained 365 downregulated and 535 upregulated genes: the $|\operatorname{logFC}|$ of the downregulated genes ranged from 1 to 5.86, and the $|\operatorname{logFC}|$ of the upregulated genes ranged from 1 to 4.4 .

\section{Construction of co-expression networks of differentially expressed genes}

Protein interactions of the expression products of the 900 differentially expressed genes were identified by calculating Pearson correlation coefficients. Based on the criterion that the absolute value of the Pearson correlation coefficient is $>0.75$, total 35 protein-protein interactions were identified for 9 normal genes and 20 differentially expressed genes (Figure 1). The 9 normal genes were PCNA (proliferating cell nuclear antigen), MCM3 (DNA replication licensing factor 3), MCM6 (DNA replication licensing factor 6), MCM7 (DNA replication licensing factor 7), ORC6L (origin recognition complex subunit 6 like), CDT1 (chromatin licensing and DNA replication factor 1), MSH2 (mutS homolog 2), FBXO5 (F-box only protein 5), and UBE2C (ubiquitin-conjugating enzyme E2C). The 20 differentially expressed genes were CCNB2 (cyclin B2), MCM10, MCM2, MCM4, CDC6 (cell division cycle 6), POLD1 (DNA 
polymerase delta catalytic subunit gene 1), CDK4 (cyclin-dependent kinase 4), KIAA0101 (PCNA-associated factor), CCNB1, PBK (PDZ-binding kinase), MYC (myelocytomatosis oncogene), SKP2 (S-phase kinase-associated protein 2 and E3 ubiquitin protein ligase), CKS1B (CDC28 protein kinase regulatory subunit 1B), CDC20, BUB1B (budding uninhibited by benzimidazoles 1 homolog beta), MAD2L1 (mitotic arrest deficient-like 1), NDC80 (kinetochore complex component homolog), AURKA (aurora kinase A), TPX2 (microtubule-associated protein homolog), and KIF11 (kinesin family member 11).

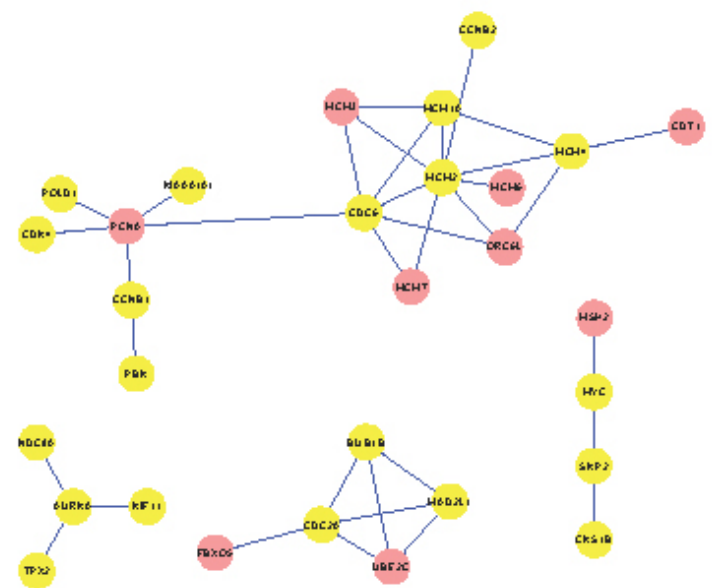

Figure 1. Co-expression network of differentially expressed genes. Yellow dots represent differentially expressed genes, pink dots represent normal genes, and lines represent protein-protein interactions.

\section{Enriched modules of differentially expressed gene in the co-expression network}

ClusterONE found enriched modules based on the protein-protein interaction network. The genes in these modules were mainly of the MCM gene family (Figure 2): MCM2, MCM3, MCM4, MCM6, MCM7, MCM10, CDC6, ORC6L, CCNB2, and CDT1. This result indicated that the MCM family plays an important role in the co-expression network.

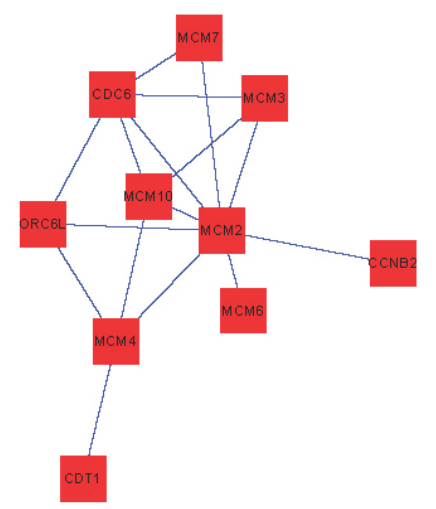

Figure 2. Co-expression network module of differentially expressed genes. We validated the co-expression network analysis and the importance of the MCM gene family had been verified. 


\section{Function-enrichment analysis of differentially expressed genes in the co-expression network}

Using the David software for biological function-enrichment analysis of differentially expressed genes based on the co-expression networks ( $\mathrm{P}<0.05$, FDR $<0.1), 10$ biological processes were identified to be enriched (Table 1). Of these 10 processes, the cell cycle was significant, including other cell cycle processes and the mitotic cell cycle. These significant function-enrichment pathways contained genes: CDC6 and CDC20.

\begin{tabular}{lccc}
\multicolumn{2}{l}{ Table 1. Enrichment analysis of differentially expressed gene function in the co-expression networks. } \\
\hline Term & Count & P value & FDR \\
\hline GO:0007049 cell cycle & 18 & $3.27 \mathrm{E}-15$ & $4.57 \mathrm{E}-12$ \\
GO:0022402 cell cycle process & 16 & $1.87 \mathrm{E}-14$ & $2.65 \mathrm{E}-11$ \\
GO:0000278 mitotic cell cycle & 14 & $5.51 \mathrm{E}-14$ & $7.84 \mathrm{E}-11$ \\
GO:0022403 cell cycle phase & 14 & $2.33 \mathrm{E}-13$ & $3.31 \mathrm{E}-10$ \\
GO:0007067 mitosis & 11 & $6.99 \mathrm{E}-12$ & $9.93 \mathrm{E}-09$ \\
GO:0000280 nuclear division & 11 & $6.99 \mathrm{E}-12$ & $9.93 \mathrm{E}-09$ \\
GO:0000087 M phase of mitotic cell cycle & 11 & $8.36 \mathrm{E}-12$ & $1.19 \mathrm{E}-08$ \\
GO:0048285 organelle fission & 11 & $1.04 \mathrm{E}-11$ & $1.48 \mathrm{E}-08$ \\
GO:0000279 M phase & 11 & $3.69 \mathrm{E}-10$ & $5.24 \mathrm{E}-07$ \\
GO:0051301 cell division & 10 & $3.28 \mathrm{E}-09$ & $4.66 \mathrm{E}-06$ \\
\hline
\end{tabular}

Terms = ID number and the name of GO; counts $=$ the number of genes involved in this process. The smaller the column P value and FDR, the stronger the enrichment.

\section{Two-way clustering analysis}

The Cheng and Church (Méndez and Stillman, 2000) algorithm (based on a mean square residual fraction) was used to perform two-way clustering. As shown in Figure 3, the volatility of MCM2 and CDC20 expression profiling was the minimum, and thus, the MCM gene family and cell cycle played important roles in the functional module (Table 2).

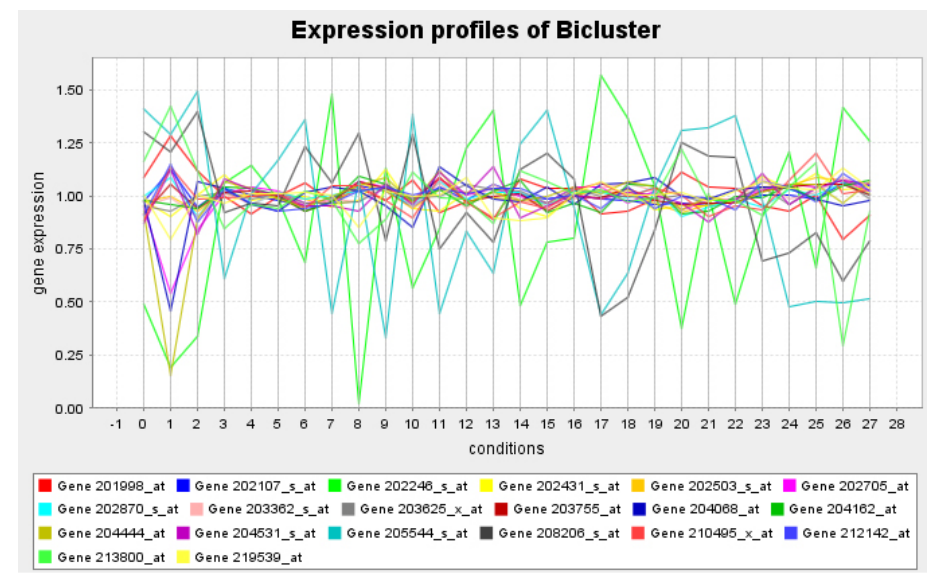

Figure 3. Visualization of two-way clustering expression profiling data. The color curve represents single gene in clusters (Gene ID and symbol table are given in Table 2). The $\mathrm{x}$-axis represents discrete conditions, the y-axis represents gene expression values. In different conditions the MCM2 and CDC20 expression profiling volatility was the minimum and we verified that MCM gene family and cell cycle played important roles in the functional module. 


\begin{tabular}{|c|c|c|c|}
\hline ID & Gene symbol & ID & Gene symbol \\
\hline 205544_s_at & CR2 & 204531_s_at & BRCA1 \\
\hline 208206_s_at & RASGRP2 & 204068_at & STK3 \\
\hline 213800_at & $\mathrm{CFH}$ & $203755^{-}$at & BUB1B \\
\hline 201998_at & ST6GAL1 & 203625 _x_at & SKP2 \\
\hline 204162_at & NDC80 & 202431_s_at & MYC \\
\hline $202246^{-} \mathrm{s}$ at & CDK4 & $203362^{-} \mathrm{s}$ at & MAD2L1 \\
\hline $202870 \mathrm{~s}$ at & CDC20 & $210495 \times$ at & FN1 \\
\hline $219539^{-}$at & GEMIN6 & $202503 \mathrm{~s}$ at & KIAA0101 \\
\hline 212142_at & MCM4 & 204444_at & KIF11 \\
\hline 202107_s_at & MCM2 & 202705_at & CCNB2 \\
\hline
\end{tabular}

\section{DISCUSSION}

Five networks of differentially expressed genes were found in the co-expressed network of differentially expressed genes, and 1 important network contained MCM2 and CDC6. The MCM2 gene belongs to the mini-chromosome maintenance (MCM) gene family and is essential for DNA replication, providing a basis for targeted treatment of NPC because the MCM protein is acting at the start point of eukaryotic genome replication (Al-Akwaa et al., 2009) in the form of a hexadimeric protein of the important pre-replication complex. MCM4, MCM6, and $\mathrm{CMC} 7$ form a protein complex that regulates helicase activity. The MCM protein is phosphorylated and regulated by protein kinases CDC2 and CDC7 (Mincheva et al., 1994). CDC7 and MCM2 expression can be used to assess tumor proliferation and may be a useful marker for predicting the outcome of DLBCL patients (Hou et al., 2011). MCM2 and MCM5 as prognostic markers of colon cancer can interact with AURKA (Burger, 2009). The MCM27 complex provides an essential replicative helicase function. MCM3 is a negative regulator of the MCM2-7 helicase in vivo by complexing with MCM5 in a manner dependent on the nuclear-export signal-like domain, blocking the recruitment of MCMs to chromatin in mammalian cells (Chuang et al., 2012). In the MCM2 co-expression network, eukaryotic protein CDC6 (part of the replication complex) is another important regulatory gene of DNA replication, maintenance of the S phase, and mitosis. CDC6 plays an important role in cell-cycle checkpoint activation and is essential for loading MCM proteins, the initiation step of DNA synthesis. Studies have shown that CDC6 has original carcinogenic activity, and overexpression of CDC6 inhibits the expression of INK/ARF (Todorov et al., 1991).

Based on our results, CDC20 was differentially expressed and belonged to another co-expression network. In vivo, CDC20 forms a complex with APC, and the APC-CDC20 protein complex has 2 main downstream targets. This protein complex prevents attachment and eventually causes adhesion loss, thus leading to the separation of sister chromatids. APCCDC20 also disrupts the cell cycle at the $\mathrm{S}$ and $\mathrm{M}(\mathrm{S} / \mathrm{M})$ phases, inactivating the $\mathrm{S} / \mathrm{M}$ phase cyclin dependent protein kinase (CDKs), leading to exit from mitosis. MCC is continuously assembled and disassembled to enable rapid activation of APC/C (CDC20), and CDC20 auto-ubiquitylation promotes MCC disassembly (Uzunova et al., 2012). At present, CDC20, a complex with 11-13 subunits, is the most important activator of anaphase-promoting complex (APC), which initiates chromatid separation. CDC20 homologue-1 (CDH1) plays a complementary role, acting as a regulatory protein in the cell cycle and interacting with many other proteins. CDC20 has 2 necessary microtubule-associated processes: anaphase chromosome 
separation and nuclear motion. The reported functions of these 2 genes are consistent with our NPC study.

PCNA is another important co-expression network in our study. A statistically significant correlation was found between PCNA and MCM2 in the tumor tissue and metastatic lymph nodes, with depth of wall invasion and local lymph node involvement (Czyzewska et al., 2009). CDK4 and PCNA can form complexes with cyclin D1 in staurosporine arrest of normal human diploid fibroblasts (Gadbois et al., 1995).

In the AURKA co-expression network, AURKA played an important role in the network according to our study. Overexpressed AURKA and AURKB present unfavorable cytogenetic abnormalities in most cases. Lucena-Araujo et al. (2011) observed a significant association between overexpression of AURKA/B and cytogenetics findings in AML, which may be relevant to new therapeutic approaches. TPX2 and AURKA affect cell viability and anchorage-independent growth, and downmodulation of TPX2 and AURKA inhibits cancer cell invasion (Sillars-Hardebol et al., 2012). Buger (2009) reported that MCM2 and MCM5 (as prognostic markers in colon cancer) can interact with AURKA (Chan et al., 2002), a finding that is supported by our study.

Based on a minimum node cluster size of 25 , we screened all genes and function modules and found that the MCM gene family was important for EBV-related NPC. Furthermore, we found that the cell cycle function module played an important role in EBV-related NPC. The processes and pathways of the cell cycle thus play important roles for the prevention and diagnosis of late-stage NPC.

The development of new biomedical techniques has enabled precise radiotherapy to combat NPC. However, the approximate 5-10-year survival rate after radiotherapy alone to treat stage III-IV NPC is only $40 \%$. Chemotherapy combined with radiotherapy improves this survival rate by decreasing the local recurrence and distant metastasis. With the development of chip technology, recently published randomized clinical studies, and meta-analysis of results (Cheng and Church, 2000), new targeted chemotherapy have led to the reduction of drug toxicity and an improved survival rate. We studied gene and function modules, and the MCM gene family was identified, providing a basis for use of targeted chemotherapy.

\section{REFERENCES}

Al-Akwaa FM, Ali MH and Kadah YM (2009). BicAT_Plus: An Automatic Comparative Tool for Bi/Clustering of Gene Expression Data Obtained Using Microarrays. Radio Science Conference. NRSC 2009. National. 2009: IEEE, New Cairo.

Bader GD and Hogue CW (2003). An automated method for finding molecular complexes in large protein interaction networks. BMC Bioinformatics 4: 2.

Barkow S, Bleuler S, Prelic A, Zimmermann P, et al. (2006). BicAT: a biclustering analysis toolbox. Bioinformatics 22: 1282-1283.

Benjamini Y and Hochberg Y (1995). Controlling the false discovery rate: a practical and powerful approach to multiple testing. J. R. Stat. Soc. B 57: 289-300.

Bose S, Yap LF, Fung M, Starzcynski J, et al. (2009). The ATM tumour suppressor gene is down-regulated in EBVassociated nasopharyngeal carcinoma. J. Pathol. 217: 345-352.

Breda E, Catarino RJ, Azevedo I, Lobao M, et al. (2010). Epstein-Barr virus detection in nasopharyngeal carcinoma: implications in a low-risk area. Braz. J. Otorhinolaryngol. 76: 310-315.

Burger M (2009). MCM2 and MCM5 as prognostic markers in colon cancer: a worthwhile approach. Dig. Dis. Sci. 54: 197-198.

Chan ATC, Teo PML, Ngan RK, Leung TW, et al. (2002). Concurrent chemotherapy-radiotherapy compared with radiotherapy alone in locoregionally advanced nasopharyngeal carcinoma: progression-free survival analysis of a phase III randomized trial. J. Clin. Oncol. 20: 2038-2044. 
Cheng Y and Church GM (2000). Biclustering of Expression Data. Proceedings of the Eighth International Conference on Intelligent Systems for Molecular Biology, Menlo Park.

Chuang CH, Yang D, Bai G, Freeland A, et al. (2012). Post-transcriptional homeostasis and regulation of MCM2-7 in mammalian cells. Nucleic Acids Res. 40: 4914-4924.

Czyzewska J, Guzinska-Ustymowicz K, Pryczynicz A, Kemona A, et al. (2009). Immunohistochemical evaluation of Ki-67, PCNA and MCM2 proteins proliferation index (PI) in advanced gastric cancer. Folia Histochem. Cytobiol. 47: 289-296.

Derrick TR, Bates BT and Dufek JS (1994). Evaluation of time-series data sets using the Pearson product-moment correlation coefficient. Med. Sci. Sports Exerc. 26: 919-928.

Fåhraeus R, Fu HL, Ernberg I, Finke J, et al. (2006). Expression of Epstein-Barr virus-encoded proteins in nasopharyngeal carcinoma. Int. J. Cancer 42: 329-338.

Fujita A, Sato JR, Rodrigues LO, Ferreira CE, et al. (2006). Evaluating different methods of microarray data normalization. BMC Bioinformatics 7: 469.

Gadbois DM, Peterson S, Bradbury EM and Lehnert BE (1995). CDK4/cyclin D1/PCNA complexes during staurosporineinduced G1 arrest and G0 arrest of human fibroblasts. Exp. Cell Res. 220: 220-225.

Hildesheim A and Wang CP (2012). Genetic predisposition factors and nasopharyngeal carcinoma risk: a review of epidemiological association studies, 2000-2011: Rosetta Stone for NPC: genetics, viral infection, and other environmental factors. Semin. Cancer Biol. 22: 107-116.

Hitt MM, Allday MJ, Hara T, Karran L, et al. (1989). EBV gene expression in an NPC-related tumour. EMBO J. 8: 26392651.

Hou Y, Wang HQ, Fu K, Zhang HL, et al. (2011). Expression of Cdc7 and mcm2 as a marker for proliferation and prognosis in diffuse large B cell lymphoma. Zhonghua Zhong Liu Za Zhi 33: 911-915.

Huang DW, Sherman BT and Lempicki RA (2009). Systematic and integrative analysis of large gene lists using DAVID bioinformatics resources. Nat. Protocol. 4: 44-57.

Keshava Prasad TS, Goel R, Kandasamy K, Keerthikumar S, et al. (2009). Human Protein Reference Database-2009 update. Nucleic Acids Res. 37: D767-D772.

Lan MY, Chen CL, Lin KT, Lee SA, et al. (2010). From NPC therapeutic target identification to potential treatment strategy. Mol. Cancer Ther. 9: 2511-2523.

Liu Y, Fang Z, Liu L, Yang S, et al. (2011). Detection of Epstein-Barr virus DNA in serum or plasma for nasopharyngeal cancer: a meta-analysis. Genet. Test. Mol. Biomarkers 15: 495-502.

Lo KW, Chung GT and To KF (2012). Deciphering the molecular genetic basis of NPC through molecular, cytogenetic, and epigenetic approaches. Semin. Cancer Biol. 22: 79-86.

Lucena-Araujo AR, de Oliveira FM, Leite-Cueva SD, dos Santos GA, et al. (2011). High expression of AURKA and AURKB is associated with unfavorable cytogenetic abnormalities and high white blood cell count in patients with acute myeloid leukemia. Leuk. Res. 35: 260-264.

Méndez J and Stillman B (2000). Chromatin association of human origin recognition complex, cdc6, and minichromosome maintenance proteins during the cell cycle: assembly of prereplication complexes in late mitosis. Mol. Cell Biol. 20: $8602-8612$.

Mincheva A, Todorov I, Werner D, Fink TM, et al. (1994). The human gene for nuclear protein BM28 (CDCL1), a new member of the early S-phase family of proteins, maps to chromosome band 3q21. Cytogenet. Cell Genet. 65: 276-277.

Raab-Traub N, Flynn K, Pearson G, Huang A, et al. (1987). The differentiated form of nasopharyngeal carcinoma contains Epstein-Barr virus DNA. Int. J. Cancer 39: 25-29.

Shannon P, Markiel A, Ozier O, Baliga NS, et al. (2003). Cytoscape: a software environment for integrated models of biomolecular interaction networks. Genome Res. 13: 2498-2504.

Shu J, He XJ and Li GC (2006). Construction of cDNA library from NPC tissue and screening of antigenic genes. Cell Mol. Immunol. 3: 53-57.

Sillars-Hardebol AH, Carvalho B, Tijssen M, Belien JA, et al. (2012). TPX2 and AURKA promote 20q amplicon-driven colorectal adenoma to carcinoma progression. Gut 61: 1568-1575.

Tao Q and Chan AT (2007). Nasopharyngeal carcinoma: molecular pathogenesis and therapeutic developments. Expert Rev. Mol. Med. 9: 1-24.

Todorov IT, Lavigne J, Sakr F, Kaneva R, et al. (1991). Nuclear matrix protein mitotin messenger RNA is expressed at constant levels during the cell cycle. Biochem. Biophys. Res. Commun. 177: 395-400.

Troyanskaya O, Cantor M, Sherlock G, Brown P, et al. (2001). Missing value estimation methods for DNA microarrays. Bioinformatics 17: 520-525.

Uzunova K, Dye BT, Schutz H, Ladurner R, et al. (2012). APC15 mediates CDC20 autoubiquitylation by APC/C(MCC) and disassembly of the mitotic checkpoint complex. Nat. Struct. Mol. Biol. 19: 1116-1123. 